\title{
Optimal Cutoff Age for Predicting Prognosis Associated with Serous Epithelial Ovarian Cancer: What is the best age cutoff?
}

Jihye Kim, MD', Youjean Chang ${ }^{\dagger}, M D$, Tae-Joong Kim, MD, PhD, Jeong-Won Lee, MD, PhD, Byoung-Gie Kim, MD, PhD, Duk-Soo

Bae, MD, PhD, Chel Hun Choi, MD, Ph.D*

Departments of Obstetrics and Gynecology, Samsung Medical Center, Sungkyunkwan University School of Medicine, Seoul 06351, Republic of Korea

\section{ABSTRACT}

Objective: Elderly age is one of the poor prognostic factors in epithelial ovarian cancer (EOC), but the optimal age cut-off is not known. The present study sought to identify the ideal age cutoff that represents a negative prognostic factor in EOC, considering the geriatric assessment.

Methods: Hazard ratios (HRs) with $p$-values were calculated using all possible age cutoffs with stage, histology, grade, optimality and comorbidities as covariates in multivariate Cox regression model. The trends of $p$-value and $H R$ by age cutoff were further evaluated in a subgroup of histology and in a The Cancer Genome Atlas (TCGA) dataset. In addition, propensity score-matching analysis using the identified age cutoff was performed.

Results: An age of 66 years was shown to be the most significant cutoff for defining old age with independent prognostic power [HR: 1.45; 95\% confidence interval (Cl): 1.04-2.03; $p=0.027]$. This result also observed with the analyses of serous histology subgroup and with the analysis of a TCGA dataset with $\mathbf{A}$ serous epithelial ovarian cancer. In survival analysis, patients aged $\geq 66$ years had significantly worse overall survival compared with younger individuals (56 months vs. 87months; $p=0.006$ ), even following propensity score matching (57 months vs. 78 months; $p=0.038$ ).

Conclusion: An age of 66 years is the best cutoff to define elderly age in serous EOC patients considering the geriatric assessment, and this information can be used in the $\mathbf{A}$ administration of individualized therapies in elderly EOC patients.

\section{INTRODUCTION}

Epithelial ovarian cancer (EOC) is primarily a disease diagnosed in postmenopausal women with a median age at diagnosis of 63 years and, approximately $70 \%$ of EOC cases and $85 \%$ of cancer-related deaths of EOC patients occur after the age of 55 years. Along with these demographic characteristics, several studies indicate that old age has an adverse prognostic effect for EOC patients, and the poor prognosis of elderly EOC patients is associated with offensive tumoral biology and less aggressive cytoreductive surgery and adjuvant chemotherapy due to frailty of elderly patients. Elderly patients are characterized by tremendous heterogeneity in terms of health status including comorbidities, geriatric syndromes, functional status and nutritional status.
Therefore, the physiologic age from comprehensive geriatric assessment (CGA) is one of the important indicators that clinicians can provide for tailored cancer treatment. Recently, more and more treatment options have been available; the tailoring of cancer treatment based on the physiologic age is needed. Even though many studies have selected conventionally the age of 65 to 80 years as a demarcation for elderly patients in EOC, the optimal age cutoff representing a poor prognostic indicator with CGA was not evaluated. This study was conducted to estimate the optimal cutoff age with CGA that can be used as a prognostic and geriatric assessment indicator in EOC patients using homogeneous populations treated with standardized surgery and chemotherapy in a single institution. Furthermore, we validated the prognostic significance of the chosen cutoff age with propensity score-matching analysis and with a dataset of The Cancer Genome Atlas (TCGA) of serous EOC.
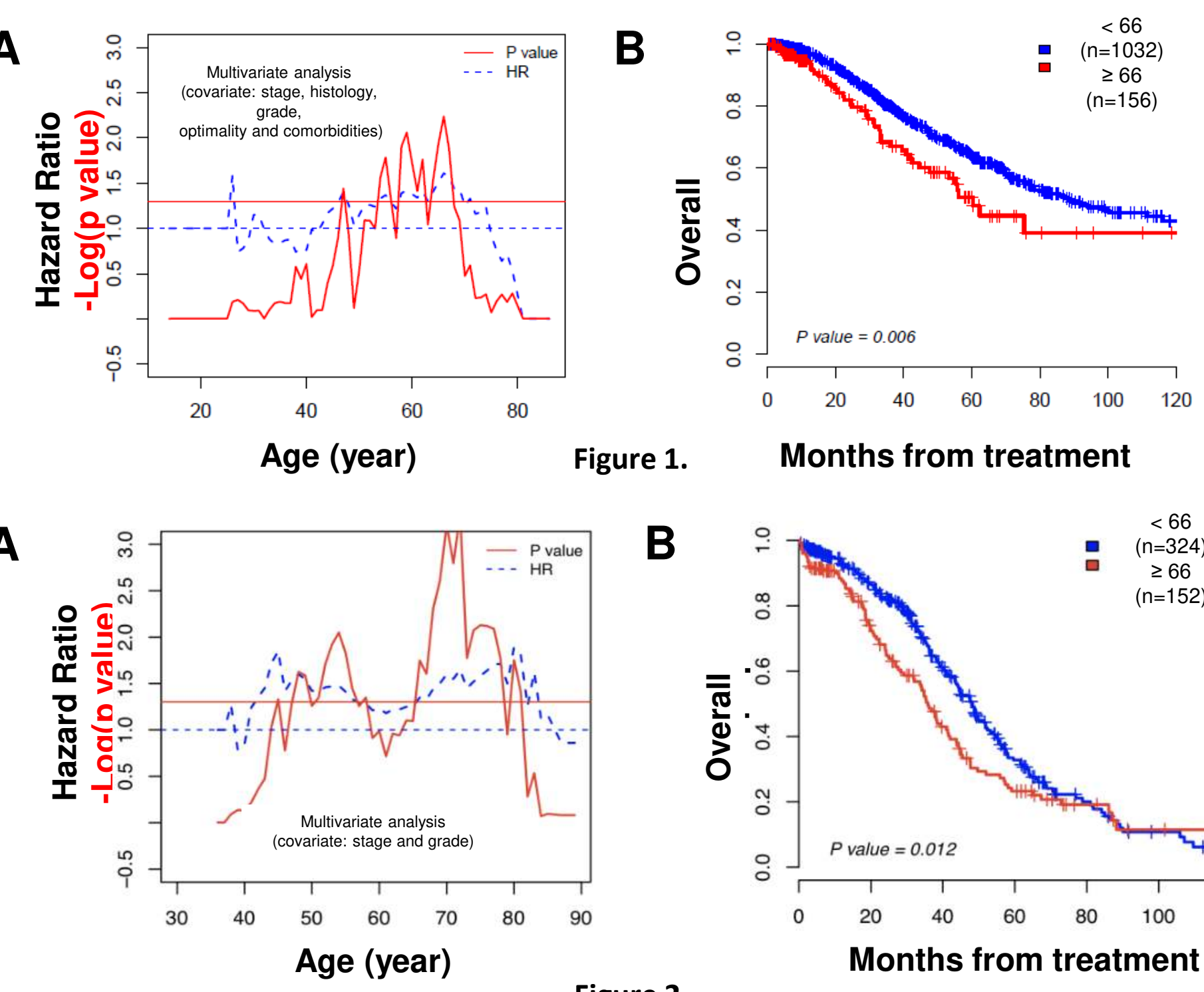

B

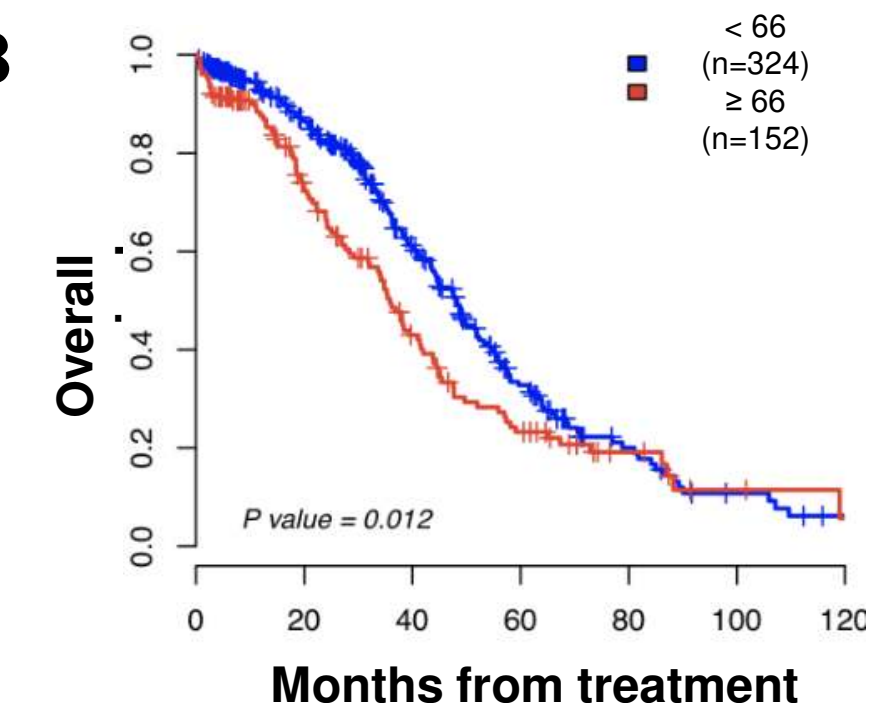

Figure 2.

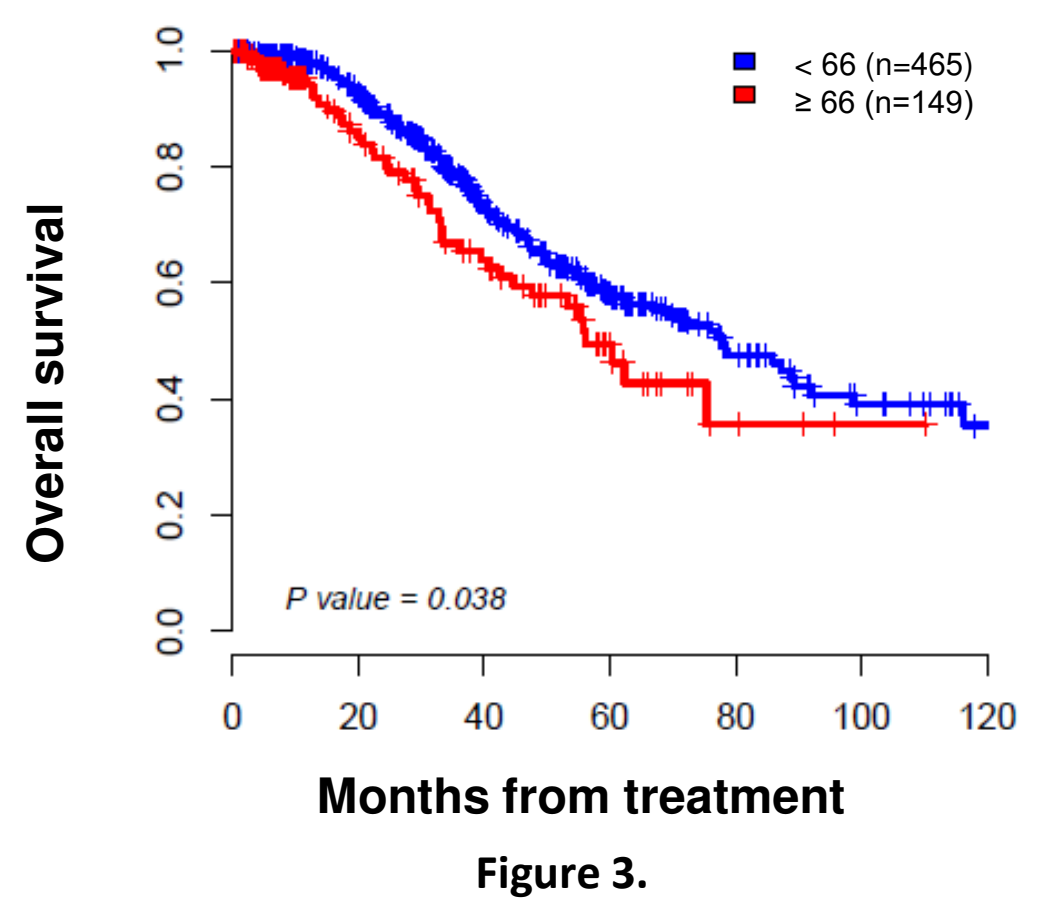

